\title{
Polychromatic electric field knots
}

\author{
Manuel F. Ferrer-Garcia ${ }^{0},{ }^{1, *}$ Alessio D’Errico $\odot,{ }^{1}$ Hugo Larocque, ${ }^{2}$ Alicia Sit, ${ }^{1}$ and Ebrahim Karimi ${ }^{1, \dagger}$ \\ ${ }^{1}$ Department of Physics, University of Ottawa, Advanced Research Complex, 25 Templeton, Ottawa, Ontario, Canada K1N 6 N5 \\ ${ }^{2}$ Research Laboratory of Electronics, Department of Electrical Engineering and Computer Science, Massachusetts Institute of Technology, \\ Cambridge, 02139 Massachusetts, USA
}

(Received 6 April 2021; accepted 10 August 2021; published 8 September 2021)

\begin{abstract}
The polarization of a monochromatic optical beam lies in a plane and, in general, is described by an ellipse, known as the polarization ellipse. The polarization ellipse in the tight-focusing (nonparaxial) regime forms nontrivial three-dimensional topologies, such as Möbius and ribbon strips as well as knots. The latter are formed when the dynamics of specifically structured polarization states are studied upon propagation. However, optical knots can also exist within another form: The electric field's tip can be made to locally oscillate along a knotted trajectory. We propose an intuitive technique to generate and engineer the path traced by the electric field vector of polychromatic beams to form different knots. In particular, we show examples of how tightly focused beams with at least three frequency components and different spatial modes can cause the tip of the electric field vector to follow, locally, a knotted trajectory. Our study may provide insight in designing current densities for structured polychromatic electromagnetic fields that interact with matter.
\end{abstract}

DOI: 10.1103/PhysRevResearch.3.033226

\section{INTRODUCTION}

The rapid advancements in the manipulation and control of electromagnetic radiation have allowed researchers to explore solutions of Maxwell's equations possessing rich topological features. The first structures that became the subject of extensive research were phase [1,2] and polarization singularities [3]. In particular, beams carrying a nonzero value of orbital angular momentum (OAM) [4] became of interest in many applications of classical and quantum optics. Optical beams with well-engineered spectra, polarization, and spatial and temporal structures are nowadays widely used in optical manipulation [5], microscopy [6-8], surface and material structuring [9], and classical and quantum communication $[10,11]$. Moreover, more complicated structures can be observed by analyzing an optical beam whose properties are not entirely defined within a two-dimensional plane, but within a three-dimensional volume. It is, indeed, predicted that the free-space trajectories of field dislocations can form closed loops $[12,13]$ with nontrivial topologies, e.g., links and knots [14]. Recently, by exploiting the technology of spatial light modulators, beams exhibiting these features have been successfully engineered in the laboratory $[13,15]$ and proposed as tools to encode prime numbers [16].

\footnotetext{
*mferr010@uottawa.ca

†ekarimi@uottawa.ca
}

Published by the American Physical Society under the terms of the Creative Commons Attribution 4.0 International license. Further distribution of this work must maintain attribution to the author $(s)$ and the published article's title, journal citation, and DOI.
Advanced tools in nonlinear optics have improved our ability to structure the frequency spectrum of an electromagnetic field, with applications such as optical manipulation and atom trapping [17-20], along with the generation of structured electric currents in semiconductors [21]. Nevertheless, the polarization states of polychromatic fields have been barely studied. While in the monochromatic case the electric field vector describes an ellipse, more complicated curves occur when multiple waves with different temporal frequencies are superimposed. This new "zoo" of polarization states, as mentioned before, remains almost unexplored; the only accurately described cases are the ones in which two fields oscillate in the same plane. If the two frequencies follow the condition $\omega_{2}=m \omega_{1}$, where $m$ is a positive integer, the local electric field traces a quartic plane curve (Lissajous-like curves) [22].

In this paper, we aim to explore the behavior of structured polychromatic fields in the nonparaxial regime, where a non-negligible component of the electric field along the propagation direction is observed. Nonparaxial fields are typically obtained by tightly focusing paraxial beams with high numerical aperture (NA) lenses [23]. It has been previously shown that, under such conditions, polarization singularities in paraxial fields can be mapped to three-dimensional structures such as Möbius strips, twisted ribbons, and skyrmionic textures [24-27]. Recently, a general scheme has been proposed in which three-dimensional superpositions of plane waves with different frequencies can create an electric field wherein its tip locally traces a knotted curve [28]. Here, we adopt the methods developed in Ref. [28] in the context of focusing by aplanatic lenses. We explore different configurations in which the polarization structure of tightly focused polychromatic beams in the form of knotted polarization oscillations is generated by means of vector diffraction 
(a)

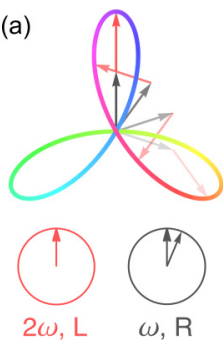

(b)

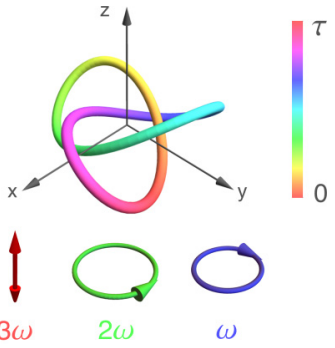

FIG. 1. (a) Paraxial polychromatic electric field (tip) tracing the planar quartic polarization curve $\mathcal{C}(t)=$ $[\cos (t)+\sin (2 t),-\sin (t)+\cos (2 t), 0]$. Fundamental $(\omega)$ and second-harmonic $(2 \omega)$ beams possess left- and right-handed polarization states, respectively. (b) Nonparaxial polychromatic electric field tracing the three-dimensional parametric curve $\mathcal{C}(t)=[\sin (3 t), \cos (t)-2 \cos (2 t), \sin (t)+2 \sin (2 t)]$. The third harmonic $(3 \omega)$ is longitudinally polarized.

(a)

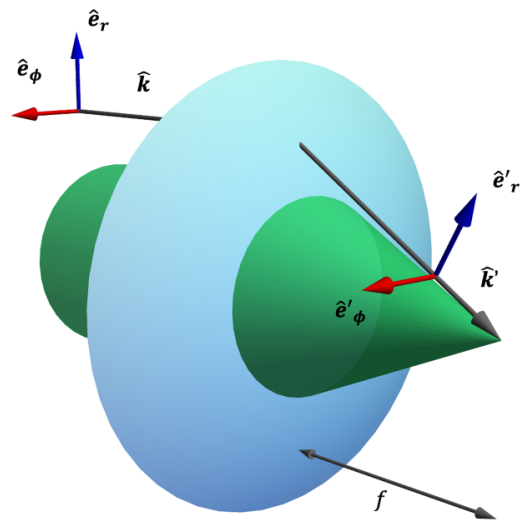

(b)
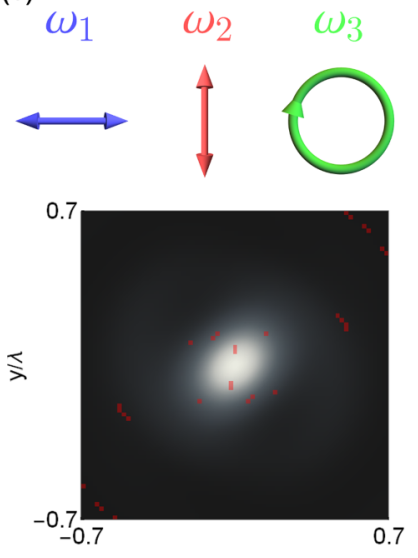

$\mathrm{x} / \lambda$ (c)
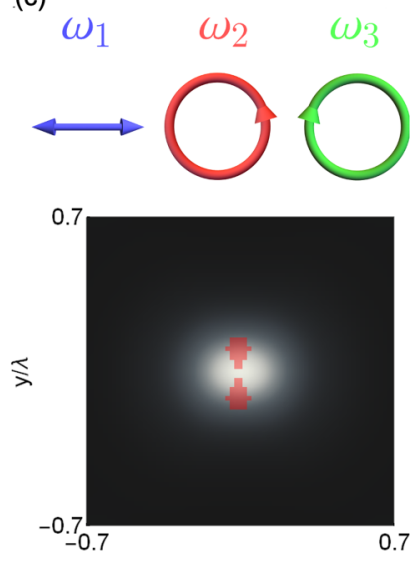

$x / \lambda$
FIG. 2. (a) Schematic diagram of the coordinate system transformation used to calculate the tightly focused fields. (b) and (c) Spatial distribution of knotted polarization curves at the focal plane obtained by tightly focusing two cases of the field given by (3), superimposed with the total intensity for $\omega_{2} / \omega_{1}=2$ and $\omega_{3} / \omega_{1}=3$ while the relative amplitudes are $a_{2} / a_{1}=a_{3} / a_{1}=2$. The red pins indicate the location of knotted polarization states. The corresponding polarization state for each frequency is shown above each panel. of polychromatic beams through an aplanatic lens. Indeed, the knotted polarizations are ubiquitous once simple conditions on the different frequencies are imposed. We follow a heuristic approach to generate large populations of knotted polarizations in the focal plane, based on the knowledge of the tight focusing of vector vortex beams. In the provided examples, we localize and identify the knotted trajectories in different planes in the focal volume.

\section{KNOTTED POLARIZATION CURVES}

In this section we briefly outline the mathematical description of knotted curves traced by time-dependent vector fields. A more detailed treatment can be found in Ref. [28]. Let us start by considering a polychromatic electric field described by the superposition of $N$ temporal Fourier components,

$$
\mathbf{E}(\mathbf{r} ; t)=\sum_{n=1}^{N} \operatorname{Re}\left[\mathbf{A}_{n}(\mathbf{r}) e^{-i \omega_{n} t}\right],
$$

where $\left\{\omega_{n}\right\}$ is the set of optical frequencies, while $\mathbf{A}_{n}$ is the local complex amplitude. For each point in space, the tip of the electric field vector describes a closed three-dimensional curve $\mathcal{C}(t)$ for $t \in\left[0,2 \pi / \omega_{p}\right]$, where $\omega_{p}$ is the greatest common divisor of the set $\omega_{n}$ (see Fig. 1). In general, it is possible to construct a set of parametric equations such that $\mathcal{C}(t)=[X(t), Y(t), Z(t)]$, where $X(t), Y(t)$, and $Z(t)$ are finite Fourier series with $i, j$, and $k$ frequency components. The
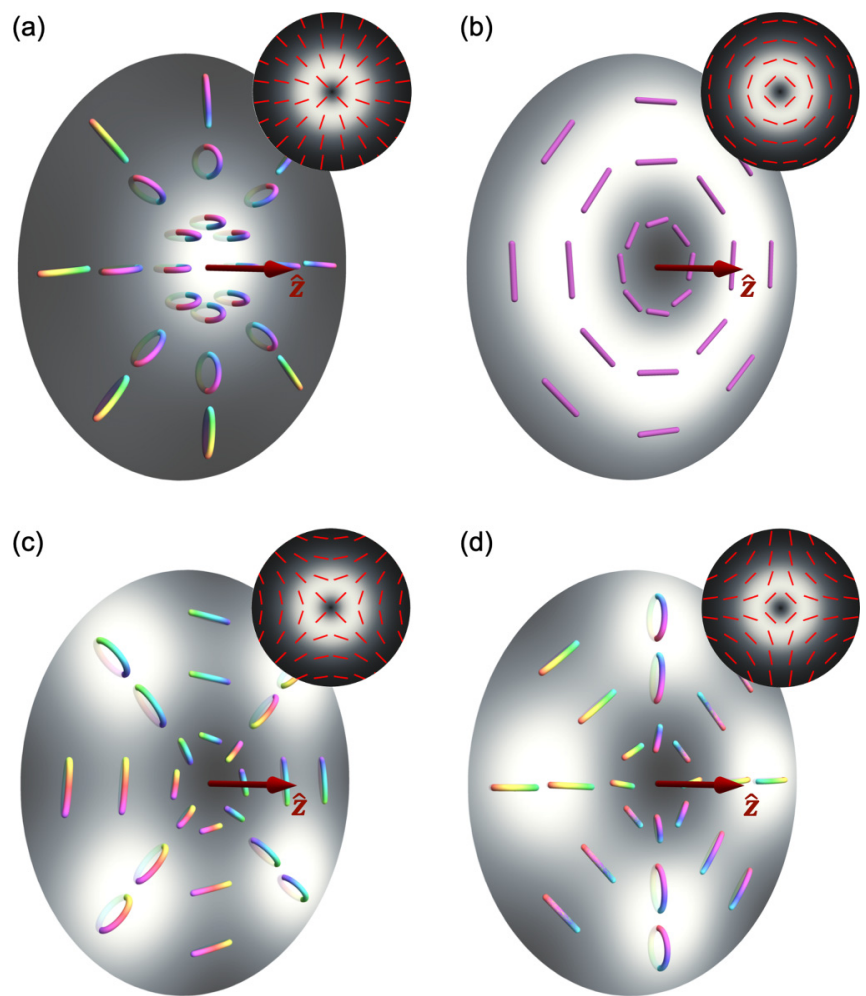

FIG. 3. Three-dimensional polarization distribution at the focal plane $z=0$ for the tightly focused vector vortex beam: (a) $\mathbf{E}_{\mathbf{v}}(\mathbf{r} ; 1,0)$, (b) $\mathbf{E}_{\mathbf{v}}(\mathbf{r} ; 1, \pi)$, (c) $\mathbf{E}_{\mathbf{v}}(\mathbf{r} ;-1,0)$, and (d) $\mathbf{E}_{\mathbf{v}}(\mathbf{r} ;-1, \pi)$. Insets show the intensity and polarization distributions of the beams in the paraxial regime. 
parametrized curve $\mathcal{C}(t)$ is defined as a Fourier- $(i, j, k)$ knot [29]. These three-dimensional curves simplify to the wellknown polarization ellipse for the case of monochromatic light $(N=1)$ and to the Lissajous-like singularities for the paraxial case, i.e., $Z(t)=0$ [22]. Similarly, a nontrivial knot is defined as a curve that cannot be transformed through smooth deformations into a simple loop, also known as the unknot. Knots can thus be classified in terms of topological invariants, i.e., mathematical objects that label sets of curves which can be smoothly transformed into each other [30]. Any transition from one type of knot to another corresponds to passing through a self-intersecting curve. For the simplest case of a Fourier-(1,1,1) knot, the local electric field must have the form

$$
\mathbf{e}=\sum_{m=1}^{3} \operatorname{Re}\left[a_{m} e^{-i \omega_{m} t} \hat{\mathbf{e}}_{\mathbf{m}}\right],
$$

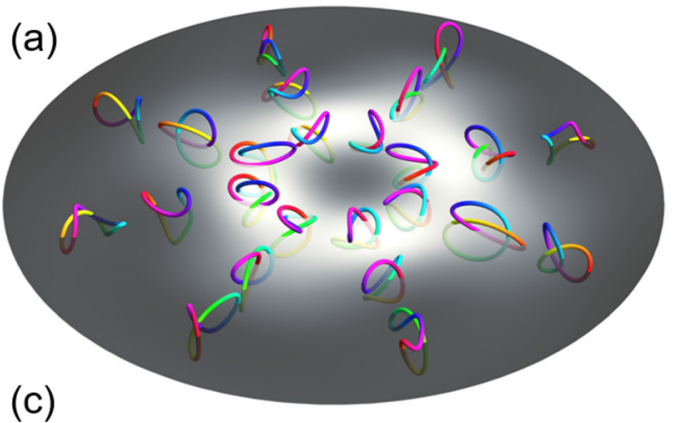

(c)

(d)
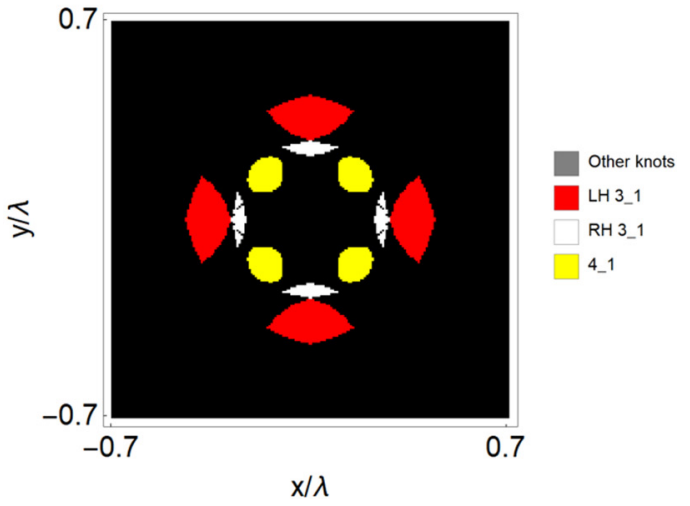

(e)

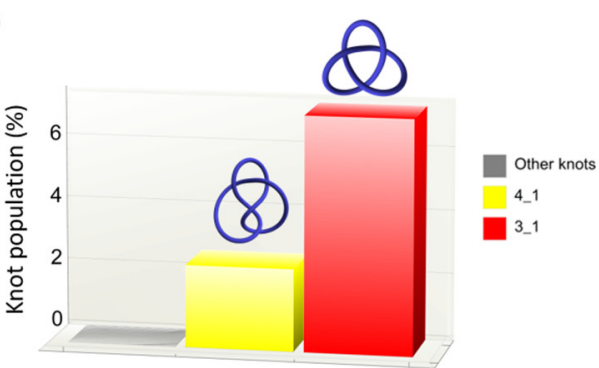

(b)

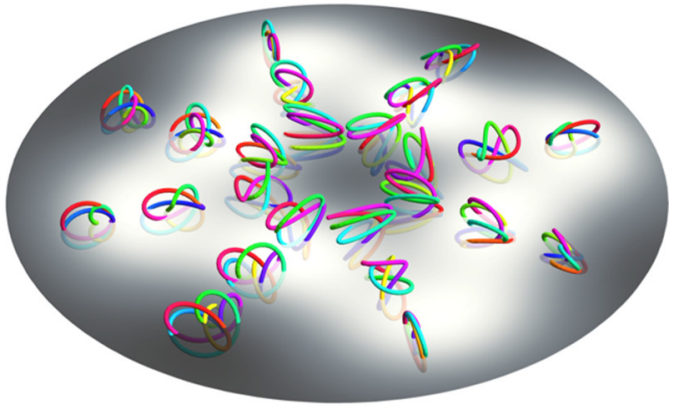

where $\left\{\hat{\mathbf{e}}_{\mathbf{m}}\right\}$ is the set of unit vectors that define a local orthogonal three-dimensional basis and $\left\{a_{m}\right\}$ are complex numbers related to the relative intensity and phase among the chromatic components. In addition, the frequencies $\left\{\omega_{m}\right\}$ must be proportional to three coprime numbers [30].

\section{KNOTTING LIGHT BY TIGHT-FOCUSING STRUCTURED POLYCHROMATIC LIGHT}

For paraxial beams, one can only create two-dimensional polarization curves (e.g., Lissajous figures); this is due to the electric field being confined in a plane orthogonal to the fixed propagation direction. However, knotted polarization curves can be achieved by interfering electric fields with different frequencies and different propagation directions; this creates the three oscillation directions needed for three-dimensional curves [28]. In contrast to direct interference, nonparaxiality can be achieved by tight focusing, where the desired dif-

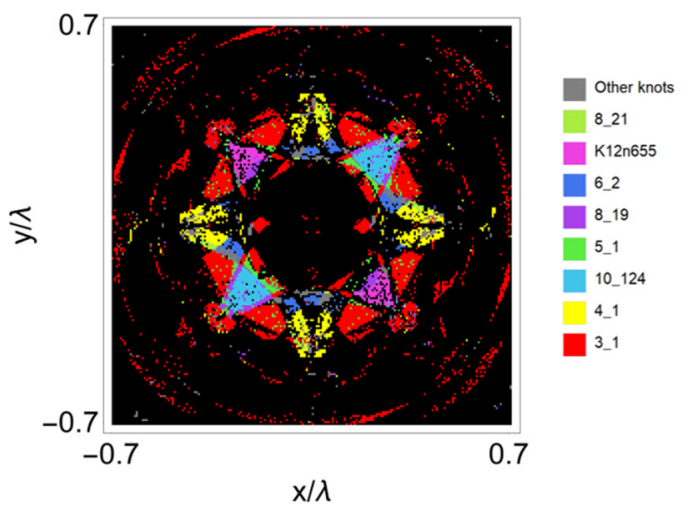

(f)

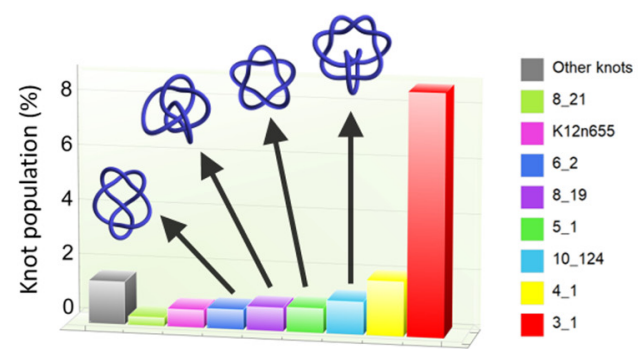

FIG. 4. Distribution of polarization curves (forming knots) at the focal plane obtained by tight-focusing the field given by (5). For (a), (c), and (e), $\omega_{2} / \omega_{1}=3$ and $\omega_{3} / \omega_{1}=2$, and for (b), (d), and (f), $\omega_{2} / \omega_{1}=3 / 2$ and $\omega_{3} / \omega_{1}=5 / 2$. In (a) and (b), some of the curves traced by the local electric field superimposed with the total intensity are shown. For (c) and (d), we performed a point-by-point classification of the knots traced by the local electric field. In (c) we also identified the chirality of the trefoil knots. Different colors correspond to different types of knots, as specified in the legend according to the standard nomenclature. LH, left handed; RH, right handed. Black is associated with unknots. The histograms (e) and (f) show the relative probability density of the different knot topologies within the considered region. 

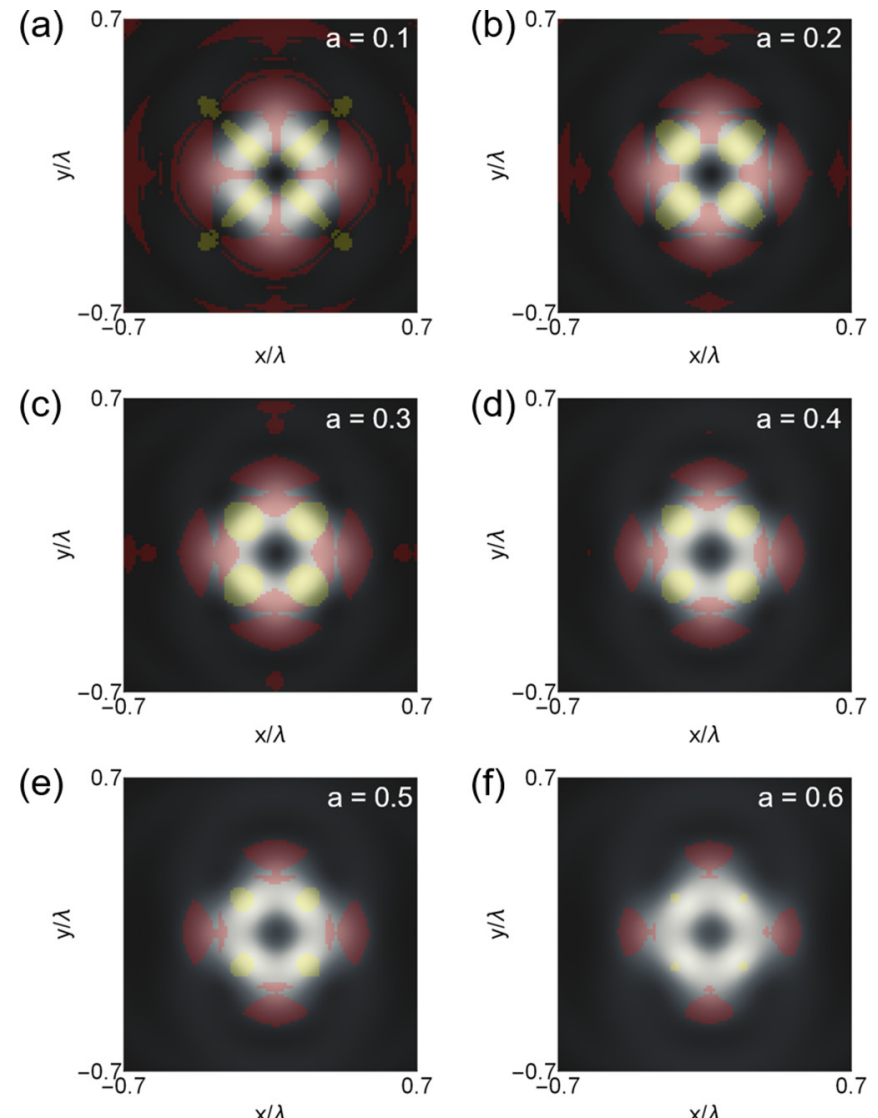

FIG. 5. Spatial distribution of polarization curves at the focal plane obtained by tight-focusing the field given by Eq. (5) when the frequencies follow the ratios $\omega_{2} / \omega_{1}=3$ and $\omega_{3} / \omega_{1}=2$ for different values of $a=a_{1} / a_{2}=a_{1} / a_{3}$. Red and yellow markers correspond to trefoil and figure-eight knotted polarizations, respectively. Meanwhile, black markers are associated with unknots.

fering propagation directions-and, consequently, all three components of the electric field-naturally arise. The timedependent fields at a plane in the focal volume are given by the the Richards-Wolf diffraction integral [23]. We calculated the components of the focused fields by numerical methods (see Appendix A). Here, we have considered that the focusing occurs in air due to a microscope objective with a high numerical aperture $(\mathrm{NA}=0.95)$. From the obtained fields, the polychromatic polarization states can be constructed by the superposition of the different harmonic components. It is noteworthy that a plethora of complicated polarization curves is expected due to the nonuniform amplitude distribution arising from the tight focusing.

We then proceed to perform the identification and classification of the polarization curves. While some knots can be recognized by visual inspection, numerical tools were employed for a more systematic approach in order to classify the three-dimensional curves [31]. A set of 10000 curves uniformly distributed within the numerical window at the plane of interest in the focal volume are considered in the classification. The identification of each curve is done by making use of the knot determinant and the Alexander polynomial, while its chirality is tested by calculating the Jones polynomial [30].

\section{RESULTS AND DISCUSSION}

As a first example, let us start by considering the simplest possible case given as the superposition of three Gaussian beams with different polarization states and frequencies. Therefore it is possible to write such a superposition as

$$
\mathbf{E}(\mathbf{r}, t)=e^{-r^{2} / w^{2}} \sum_{m=1}^{3} a_{m} e^{-i \omega_{m} t} \hat{\mathbf{e}}_{\mathbf{m}}
$$

where $w$ stands for the waist parameter of a Gaussian beam, which we assumed to be equal for all three harmonic components. Figures 2(b) and 2(c) show two possible configurations for the electric field described by Eq. (3) where the frequencies are related by $\omega_{2} / \omega_{1}=2$ and $\omega_{3} / \omega_{1}=3$, corresponding to the first three coprime numbers. Knotted polarization states are present in both cases, but the populations of these exotic states are relatively small and only contain trefoil topologies. As expected, the spatial distribution of the knotted curves on the focal plane depends on the polarization states of the paraxial beams, i.e., larger regions are obtained when the beams with higher frequencies are circularly polarized.

Though we have been able to generate knotted polarization curves, their population is small and confined to extremely localized regions. In order to develop a strategy to generate larger populations of knotted states, it is convenient to recall the action of the lens on the input beam's polarization state. As shown in Fig. 2(a), the incident polarization state is decomposed using the triad $\left\{\mathbf{e}_{r}, \mathbf{e}_{\phi}, \mathbf{k}\right\}$ [23], representing the radial, azimuthal, and wave-vector unit vectors, respectively. In this scenario, a large longitudinal component of the focused field is generated from the radial component of the input field. This component is therefore instrumental in adding a third dimension to the oscillation of the electric field, which, as shown in Eq. (1), is necessary for generating a knotted curve. Meanwhile, the azimuthal contribution of the field remains practically unchanged. These features thereby motivate expressing the three frequency components in terms of beams that predominantly polarized along $\mathbf{e}_{r}$ and $\mathbf{e}_{\phi}$. For this reason, we opt to examine the three-dimensional patterns that arise from the tight focusing of vector vortex beams. In particular, we consider the class of vector vortex beams, in cylindrical coordinates $\mathbf{r}=(r, \phi, z)$ (at the lens input pupil plane), defined as

$$
\mathbf{E}_{\mathbf{v}}(\mathbf{r} ; \ell, \delta)=\frac{1}{\sqrt{2}} A_{|\ell|}(r)\left[e^{i \ell \phi} \hat{\mathbf{e}}_{\mathbf{R}}+e^{i \delta} e^{-i \ell \phi} \hat{\mathbf{e}}_{\mathbf{L}}\right]
$$

Here, $\left\{\hat{\mathbf{e}}_{\mathbf{L}}, \hat{\mathbf{e}}_{\mathbf{R}}\right\}$ are unit vectors for left- and right-handed circular polarization; the factors $\exp ( \pm i \ell \phi)$, with $\ell$ being an integer, give the OAM content of each circular polarization component; and $A_{|\ell|}(r)$ specifies the complex radial amplitude distribution of the electric field. In our calculations, we considered the case of Laguerre-Gaussian modes, $A_{|\ell|}(\mathbf{r}) e^{i \ell \phi}=$ $\mathrm{LG}_{\ell}^{0}(r, \phi)$, with radial index $p=0$ and topological charge $\ell$. The three-dimensional polarization distributions at the focal plane $z=0$ are shown in Fig. 3 for the cases of $|\ell|=1$ and 
(a)

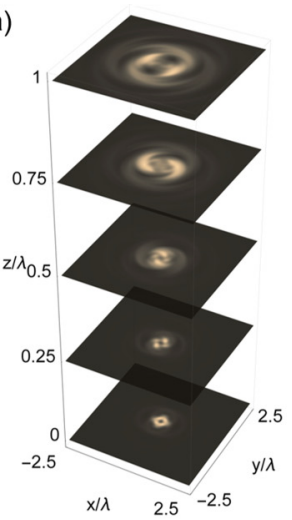

(b)

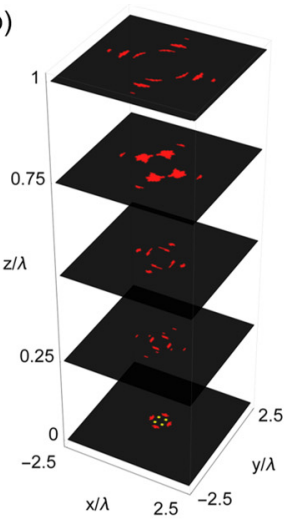

(c)

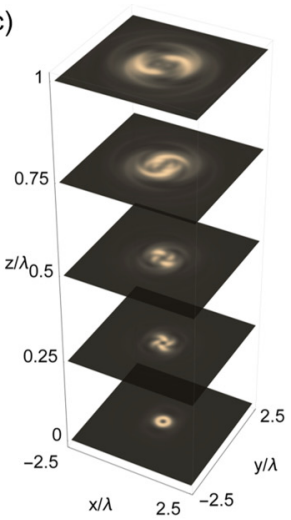

(d)

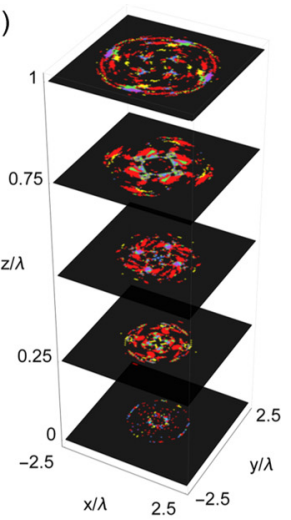

FIG. 6. Effect of propagation on total intensity and knotted polarization distribution for (a) and (b) $\omega_{2} / \omega_{1}=3$ and $\omega_{3} / \omega_{1}=2$ and (c) and (d) $\omega_{2} / \omega_{1}=3 / 2$ and $\omega_{3} / \omega_{1}=5 / 2$. Panels (a) and (c) show the total intensity distribution, while (b) and (d) display the knot positions and their types. The color coding in the knot distribution plots is the same as given in Fig. 4.

$\delta=\{0, \pi\}$ in (4). Note that, by considering a polychromatic superposition, i.e.,

$$
\begin{aligned}
\mathbf{E}(\mathbf{r}, t)= & a_{1} e^{-i \omega_{1} t} \mathbf{E}_{\mathbf{v}}(\mathbf{r}, 1,0)+a_{2} e^{-i \omega_{2} t} \mathbf{E}_{\mathbf{v}}(\mathbf{r},-1,0) \\
& +a_{3} e^{-i \omega_{3} t} \mathbf{E}_{\mathbf{v}}(\mathbf{r},-1, \pi),
\end{aligned}
$$

and focusing it, the curve traced by the local electric field vector takes the form of a Fourier- $(3,3,3)$ knot in its more general form. Additionally, in order to maximize the region in which the longitudinal component of the field is non-negligible, we set $\omega_{1}=\min \left(\left\{\omega_{n}\right\}\right)$. This condition arises from the fact that at higher frequencies, the region where the beam amplitude is non-negligible becomes smaller (see Appendix A). Therefore knotted electric field curves are formed at the focal plane depending on the chosen temporal frequencies and the local relative phase and amplitudes of the chromatic components of the input beam.

As a first example of the proposed approach, let us consider the temporal frequencies in Eq. (5) to be related by $\omega_{2} / \omega_{1}=3$ and $\omega_{3} / \omega_{1}=2$, which correspond to the first three coprime numbers. In addition, we set $a_{2} / a_{1}=a_{3} / a_{1}=2$ and consider the same waist parameter for the Laguerre-Gaussian modes. Figure 4(a) shows the the amplitude profile of the tightly focused field overlaid with the curves traced out by the resulting knotted polarization vectors. Figure 4(c) shows the regions in which different knotted structures are located on the focal plane. About $7.5 \%$ of the analyzed trajectories were identified as either left- or right-chiral trefoil knots (3_1), while $2.5 \%$ corresponded to figure-eight knots (4_1) [see Fig. 4(e)]. It should be noted that, by following a circular path around the origin, it is possible to observe the transition between unknots and knotted trajectories. It is worthy of emphasis that only the non-negligible longitudinal component arises from focusing $\mathbf{E}_{\mathbf{v}}(\mathbf{r}, 1,0)$, while the remaining terms generate the transverse basis on the focal volume. Therefore we present a brief exploration on the effect of the relative amplitude of the longitudinal component. Figure 5 depicts the spatial distribution of knotted polarization states when the ratio between the relative amplitudes $a=a_{1} / a_{2}=a_{1} / a_{3}$ is varied. In all the cases, only trefoils and figure-eight polarization knots are created. From these results, it becomes evident that the spatial distribution of the knotted polarization curves can be tailored by modifying the relative amplitude of the radial component of the superposition.

Similarly, we present the analysis of a case in which the superposition is composed of structured beams with higher harmonics of an arbitrary frequency. For instance, we consider the frequencies $\omega_{2} / \omega_{1}=3 / 2$ and $\omega_{3} / \omega_{1}=5 / 2$, where these values correspond to the next three lowest possible coprime numbers: 2, 3, and 5. As shown in Figs. 4(b), 4(d), and 4(f), about $25 \%$ of the analyzed trajectories are nontrivial knots, including the presence of trefoil, cinquefoil (5_1), and threetwist knots.

Heretofore, we have considered the existence of knotted polarization states exclusively on the focal plane $(z=0)$. A more complete analysis should include the evolution of the three-dimensional polarization distribution upon propagation in the focal volume. As shown in Fig. 6, knotted structures adiabatically follow the beam propagation. In Fig. 6(a), trefoil knots are present in a region that extends beyond the fundamental wavelength $\lambda=2 \pi c / \omega_{\min }$. Our calculations show that, since the longitudinal component of the electric field becomes negligible far from the focus, these polarization knots disappear at a distance $\Delta z=2 \lambda$ from the focal plane (not shown in the figure). Similarly, Fig. 6(b) shows the knot propagation for the case $\omega_{2} / \omega_{1}=3 / 2$ and $\omega_{3} / \omega_{1}=5 / 2$.

In summary, we have introduced a heuristic criterion to generate large and rich populations of locally knotted polarization curves by tight-focusing three structured beams at different frequencies. These structures can be generated and detected with the current techniques developed in nonlinear optics and nanophotonics, e.g., exploiting the recent advances in phase locking and independent manipulation of different harmonics [32] and, for what concerns the detection, by properly analyzing the scattering of the focused beams from metal nanoparticles [33]. In addition, interaction of these locally knotted fields with (semiconductor) materials might induce local three-dimensional magnetic behavior.

\section{ACKNOWLEDGMENTS}

The authors acknowledge the fruitful conversations with Dr. Sergey Nechayev. This work was supported by Canada Research Chairs (CRC), Canada First Excellence Research Fund (CFREF), and Ontario's Early Researcher Award. 
(a)
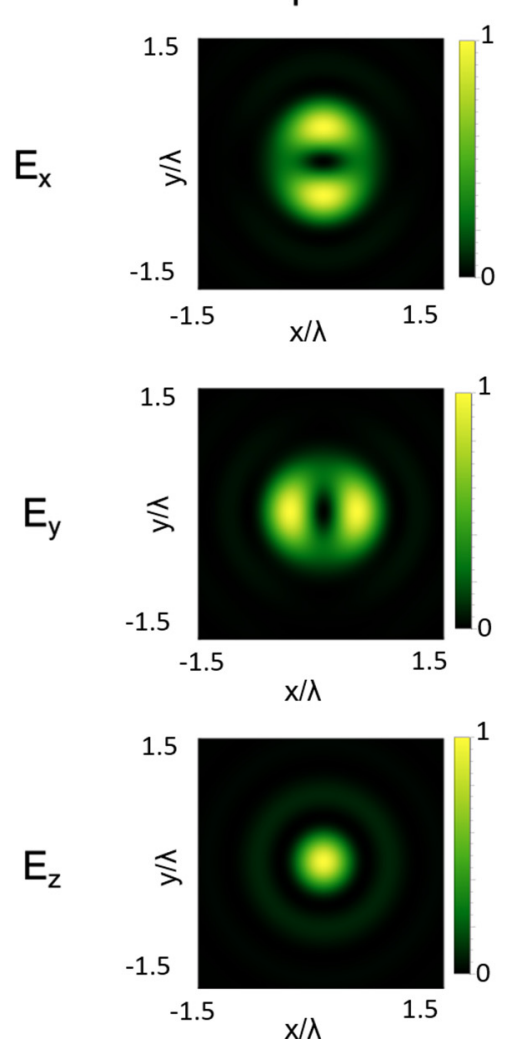

(b)
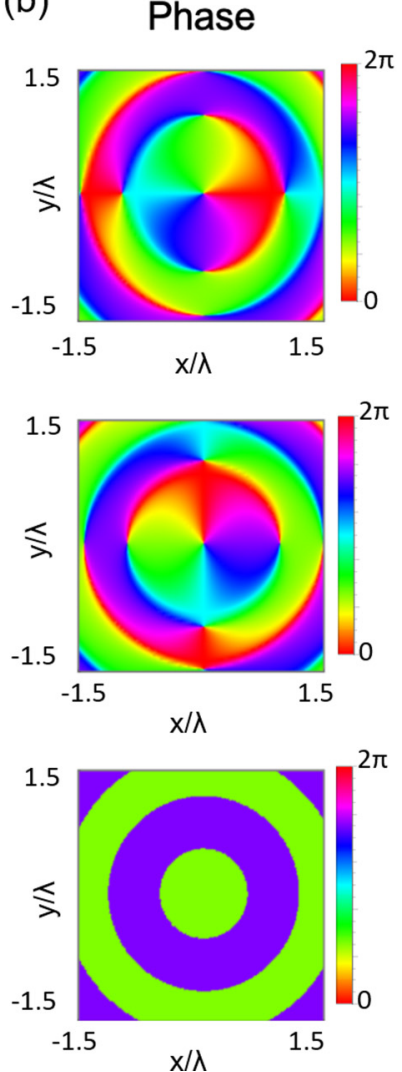

(c) Amplitude
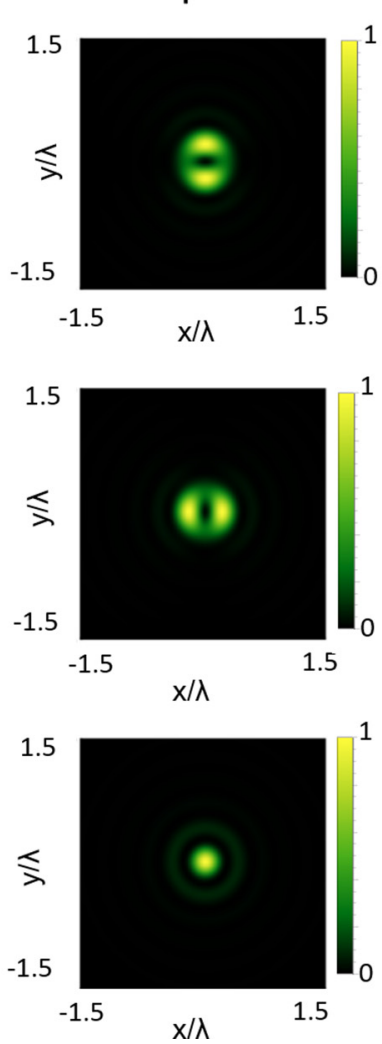

(d)

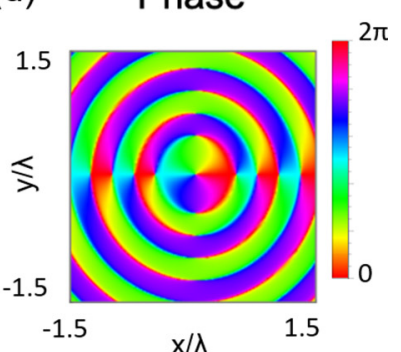

FIG. 7. Amplitude and phase distributions of the electric field components obtained by tightly focusing a LG G $_{1}^{0}$ ode (with left circular polarization). (a) and (b), and (c) and (d), correspond to two different optical frequencies: (a) and (b) $\omega_{1}=2 \pi c / \lambda$; (c) and (d) $\omega_{2}=2 \omega_{1}$.

\section{APPENDIX A: TIGHTLY FOCUSED \\ POLYCHROMATIC LIGHT}

The time-dependent electric field $\mathbf{E}_{f}(\rho, \varphi, z, t ; \omega)$ at the focal plane of an aplanatic lens, with focal length $f$ and a high numerical aperture (NA), is given by Richards and Wolf's diffraction integral [23],

$$
\mathbf{E}_{f}(\rho, \varphi, z, t ; \omega)=-\frac{i f \omega}{2 \pi c} e^{i \omega t} \int_{0}^{\Theta} \sin \theta d \theta e^{i k z \cos \theta} \int_{0}^{2 \pi} \mathbf{A}(\phi, \theta, t ; \omega) e^{i k \rho \sin \theta \cos (\varphi-\phi)} d \phi,
$$

where $k=\omega / c$ stands for the wave number, $\Theta=\arcsin (\mathrm{NA} / n)$ is the maximum angular aperture of the objective, $n$ is the refractive index of the medium, and

$$
\mathbf{A}(\phi, \theta)=\mathbf{T} \cdot \mathbf{E}_{\text {in }}(\phi, \theta)
$$

is the transformation of the initial beam $\mathbf{E}_{\text {in }}=\left(U_{x}, U_{y}, 0\right)^{T}$ after the objective. The transformation matrix is given as

$$
\mathbf{T}=\sqrt{\cos \theta}\left[\begin{array}{ccc}
\cos \theta \cos ^{2} \phi+\sin ^{2} \phi & (\cos \theta-1) \sin \phi \cos \phi & \sin \theta \cos \phi \\
(\cos \theta-1) \sin \phi \cos \phi & \cos \theta \sin ^{2} \phi+\cos ^{2} \phi & \sin \theta \sin \phi \\
-\sin \theta \cos \phi & -\sin \theta \sin \phi & \cos \theta
\end{array}\right] .
$$

Similarly, it is possible to rewrite (A1) to be

$$
\begin{aligned}
\mathbf{E}_{f}(\rho, \varphi, z)= & -\frac{i f \omega}{2 \pi c} e^{i \omega t} \sum_{m=0}^{\infty} \int_{0}^{\theta} \sin \theta d \theta e^{i k z \cos \theta} \\
& \times \int_{0}^{2 \pi} \mathbf{A}(\phi, \theta) i^{m} \mathbf{J}_{m}(k \rho \sin \theta) e^{i m(\varphi-\phi)} d \phi,
\end{aligned}
$$

where $\mathbf{J}_{m}(\cdot)$ stands for the $m$ th-order Bessel function of the first kind. Therefore it is possible to construct a tightly focused polychromatic field as

$$
\mathbf{E}(\rho, \varphi, z, t)=\sum_{m=1}^{N} \operatorname{Re}\left[a_{m} \mathbf{E}_{f}^{m}\left(\rho, \varphi, z, t ; \omega_{m}\right)\right],
$$

where $\left\{\omega_{m}\right\}$ are complex numbers. We expect to find polarization knots in regions where the intensities of the three frequency components are comparable. 
(a)

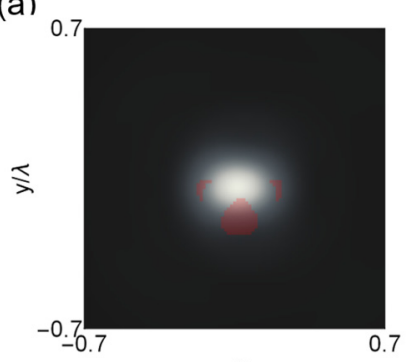

(c)

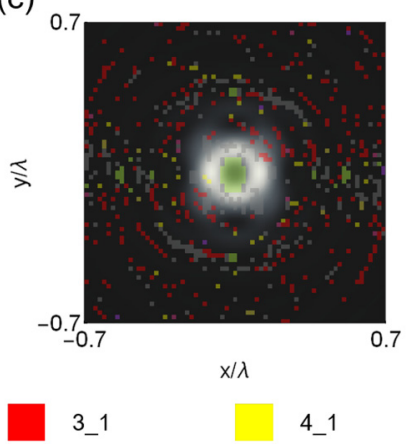

(b)

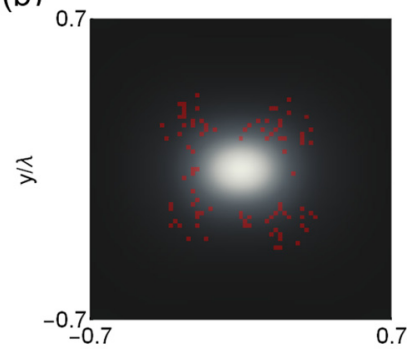

$\mathrm{x} / \lambda$

(d)

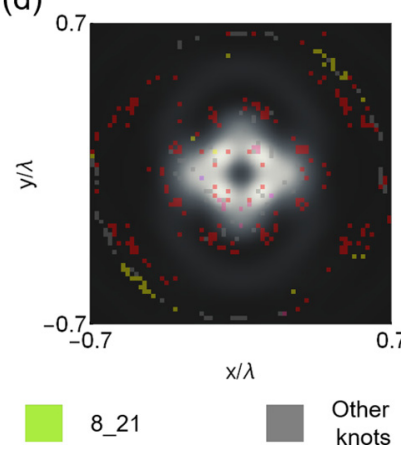

FIG. 8. Spatial distribution of knotted polarization curves at the focal plane obtained by tightly focusing the fields given by (a) Eq. (B1) for $\omega_{2} / \omega_{1}=2$ or $\omega_{3} / \omega_{1}=3$ when the Gaussian beams are circularly polarized and (b)-(d) Eq. (5) for $\omega_{2} / \omega_{1}=2$ and $\omega_{3} / \omega_{1}=3(\mathrm{~b}), \omega_{2} / \omega_{1}=5$ and $\omega_{3} / \omega_{1}=3(\mathrm{c})$, and $\omega_{2} / \omega_{1}=4 / 3$ and $\omega_{3} / \omega_{1}=5 / 3(\mathrm{~d})$.

It is thus useful to keep in mind that the spatial extension of a tightly focused beam decreases with the frequency, as illustrated in Fig. 7.

\section{APPENDIX B: OTHER KNOT PATTERNS OBTAINED FROM DIFFERENT OPTICAL SUPERPOSITIONS}

Following the analysis in Sec. IV, we present some other possible configurations. First, let us consider the superposition of two Gaussian beams with different polarization states and a radially polarized beam,

$$
\begin{aligned}
\mathbf{E}(\mathbf{r}, t)= & 2 e^{-r^{2} / w^{2}}\left(\hat{\mathbf{e}}_{\mathbf{1}} e^{-\omega_{2} t}+\hat{\mathbf{e}}_{2} e^{-\omega_{3} t}\right) \\
& +e^{-\omega_{1} t} \mathbf{E}_{\mathbf{v}}(\mathbf{r}, 1,0),
\end{aligned}
$$

where $\left\{\hat{\mathbf{e}}_{\mathbf{1}}, \hat{\mathbf{e}}_{\mathbf{1}}\right\}$ are orthogonal polarization states. For the sake of simplicity, the temporal frequencies are related by $\omega_{2} / \omega_{1}=$ 2 or $\omega_{3} / \omega_{1}=3$. Figure 8 (a) the knot distribution resulting from the tight focusing of Eq. (B1) when $\left\{\hat{\mathbf{e}}_{\mathbf{1}}, \hat{\mathbf{e}}_{\mathbf{1}}\right\}$ form the circular polarization basis. As in the case of the superposition of three Gaussian beams, a small population of knotted polarization states is generated. Meanwhile, nontrivial polarization curves are obtained when the Gaussian beams are linearly polarized. Figures 8(b)-8(d) depict the spatial distribution of three-dimensional curves obtained by tight-focusing the beam given in Eq. (5) for different sets of frequencies.
[1] J. F. Nye and M. V. Berry, Dislocations in wave trains, Proc. R. Soc. London, Ser. A 336, 165 (1974).

[2] M. Soskin, V. Gorshkov, M. Vasnetsov, J. Malos, and N. Heckenberg, Topological charge and angular momentum of light beams carrying optical vortices, Phys. Rev. A 56, 4064 (1997).

[3] M. R. Dennis, K. O’Holleran, and M. J. Padgett, Singular optics: Optical vortices and polarization singularities, Prog. Opt. 53, 293 (2009).

[4] L. Allen, M. W. Beijersbergen, R. Spreeuw, and J. Woerdman, Orbital angular momentum of light and the transformation of Laguerre-Gaussian laser modes, Phys. Rev. A 45, 8185 (1992).

[5] H. He, M. Friese, N. Heckenberg, and H. Rubinsztein-Dunlop, Direct Observation of Transfer of Angular Momentum to Absorptive Particles from a Laser Beam with a Phase Singularity, Phys. Rev. Lett. 75, 826 (1995).

[6] R. Chen, K. Agarwal, C. J. Sheppard, and X. Chen, Imaging using cylindrical vector beams in a high-numerical-aperture microscopy system, Opt. Lett. 38, 3111 (2013).

[7] K. Zhanghao, X. Chen, W. Liu, M. Li, Y. Liu, Y. Wang, S. Luo, X. Wang, C. Shan, H. Xie, J. Gao, X. Chen, D. Jin, X. Li, Y. Zhang, Q. Dai, and P. Xi, Super-resolution imaging of fluorescent dipoles via polarized structured illumination microscopy, Nat. Commun. 10, 4694 (2019).

[8] S. W. Hell and J. Wichmann, Breaking the diffraction resolution limit by stimulated emission: Stimulated-emissiondepletion fluorescence microscopy, Opt. Lett. 19, 780 (1994).
[9] J. J. Nivas, S. He, A. Rubano, A. Vecchione, D. Paparo, L. Marrucci, R. Bruzzese, and S. Amoruso, Direct femtosecond laser surface structuring with optical vortex beams generated by a q-plate, Sci. Rep. 5, 17929 (2015).

[10] A. E. Willner, H. Huang, Y. Yan, Y. Ren, N. Ahmed, G. Xie, C. Bao, L. Li, Y. Cao, Z. Zhao, J. Wang, M. P. J. Lavery, M. Tur, S. Ramachandran, A. F. Molisch, N. Ashrafi, and S. Ashrafi, Optical communications using orbital angular momentum beams, Adv. Opt. Photonics 7, 66 (2015).

[11] F. Bouchard, A. Sit, F. Hufnagel, A. Abbas, Y. Zhang, K. Heshami, R. Fickler, C. Marquardt, G. Leuchs, R. W. Boyd, and E. Karimi, Quantum cryptography with twisted photons through an outdoor underwater channel, Opt. Express 26, 22563 (2018).

[12] M. V. Berry and M. R. Dennis, Knotted and linked phase singularities in monochromatic waves, Proc. R. Soc. London, Ser. A 457, 2251 (2001).

[13] M. R. Dennis, R. P. King, B. Jack, K. O'Holleran, and M. J. Padgett, Isolated optical vortex knots, Nat. Phys. 6, 118 (2010).

[14] D. Sugic and M. R. Dennis, Singular knot bundle in light, J. Opt. Soc. Am. A 35, 1987 (2018).

[15] H. Larocque, D. Sugic, D. Mortimer, A. J. Taylor, R. Fickler, R. W. Boyd, M. R. Dennis, and E. Karimi, Reconstructing the topology of optical polarization knots, Nat. Phys. 14, 1079 (2018).

[16] H. Larocque, A. D’Errico, M. F. Ferrer-Garcia, A. Carmi, E. Cohen, and E. Karimi, Optical framed knots as information carriers, Nat. Commun. 11, 5119 (2020). 
[17] M. Feng, Quantum computing in cavity QED with cold trapped ions by bichromatic radiation, Phys. Rev. A 65, 064301 (2002).

[18] M. Andersen, C. Ryu, P. Cladé, V. Natarajan, A. Vaziri, K. Helmerson, and W. D. Phillips, Quantized Rotation of Atoms from Photons with Orbital Angular Momentum, Phys. Rev. Lett. 97, 170406 (2006).

[19] M. Bhattacharya, H. Uys, and P. Meystre, Optomechanical trapping and cooling of partially reflective mirrors, Phys. Rev. A 77, 033819 (2008).

[20] I. Kozyryev, L. Baum, L. Aldridge, P. Yu, E. E. Eyler, and J. M. Doyle, Coherent Bichromatic Force Deflection of Molecules, Phys. Rev. Lett. 120, 063205 (2018).

[21] S. Sederberg, F. Kong, F. Hufnagel, C. Zhang, E. Karimi, and P. B. Corkum, Vectorized optoelectronic control and metrology in a semiconductor, Nat. Photonics 14, 680 (2020).

[22] I. Freund, Bichromatic optical Lissajous fields, Opt. Commun. 226, 351 (2003).

[23] B. Richards and E. Wolf, Electromagnetic diffraction in optical systems, II. Structure of the image field in an aplanatic system, Proc. R. Soc. London, Ser. A 253, 358 (1959).

[24] T. Bauer, P. Banzer, E. Karimi, S. Orlov, A. Rubano, L. Marrucci, E. Santamato, R. W. Boyd, and G. Leuchs, Observation of optical polarization Möbius strips, Science 347, 964 (2015).

[25] T. Bauer, P. Banzer, F. Bouchard, S. Orlov, L. Marrucci, E. Santamato, R. W. Boyd, E. Karimi, and G. Leuchs, Multi-twist polarization ribbon topologies in highly-confined optical fields, New J. Phys. 21, 053020 (2019).

[26] T. Bauer, S. N. Khonina, I. Golub, G. Leuchs, and P. Banzer, Ultrafast spinning twisted ribbons of confined electric fields, Optica 7, 1228 (2020).

[27] S. Gao, F. C. Speirits, F. Castellucci, S. Franke-Arnold, S. M. Barnett, and J. B. Götte, Paraxial skyrmionic beams, Phys. Rev. A 102, 053513 (2020).

[28] D. Sugic, M. R. Dennis, F. Nori, and K. Y. Bliokh, Knotted polarizations and spin in three-dimensional polychromatic waves, Phys. Rev. Research 2, 042045(R) (2020).

[29] A. Stasiak and V. Katritch, Ideal Knots, Series on Knots and Everything Vol. 19 (World Scientific, Singapore, 1998).

[30] L. H. Kauffman, Knots and Physics, Series on Knots and Everything Vol. 1 (World Scientific, Singapore, 2001).

[31] A. J. Taylor and other SPOCK contributors, pyknotid knot identification toolkit, https://github.com/SPOCKnots/pyknotid (2017), accessed Dec. 1, 2020.

[32] H.-S. Chan, Z.-M. Hsieh, W.-H. Liang, A. Kung, C.-K. Lee, C.-J. Lai, R.-P. Pan, and L.-H. Peng, Synthesis and measurement of ultrafast waveforms from five discrete optical harmonics, Science 331, 1165 (2011).

[33] T. Bauer, S. Orlov, U. Peschel, P. Banzer, and G. Leuchs, Nanointerferometric amplitude and phase reconstruction of tightly focused vector beams, Nat. Photonics 8, 23 (2014). 